\title{
Gestão do conhecimento científico: proposta de um modelo conceitual com base em processos de comunicação científica
}

\author{
Fernando César Lima Leite \\ Pesquisador colaborador da Universidade de Brasília. \\ Embrapa Informação Tecnológica. \\ E-mail: fernandodfc@gmail.com

\section{Sely Maria de Souza Costa \\ $\mathrm{PhD}$ em ciência da informação. Professora da Universidade de Brasília. E-mail: selmar@unb.br}

\section{Resumo}

A gestão do conhecimento tem se desenvolvido no ambiente das organizações empresariais e tem suas pesquisas e aplicações voltadas para a perspectiva do conhecimento organizacional. No entanto, existem outros contextos nos quais a gestão do conhecimento pode ser estudada, como, por exemplo, o contexto acadêmico, voltado para a perspectiva do conhecimento científico. Independentemente do contexto em que se inserem, os processos de comunicação constituem uma questão fundamental a ser levada em consideração em estudos sobre gestão do conhecimento. Esta pesquisa se propôs a investigar a relação, em nível conceitual, entre a gestão do conhecimento e os processos de comunicação científica, tendo em vista as peculiaridades do contexto e do conhecimento científico. Mais especificamente, referiu-se a uma proposta teórica de construção de um modelo conceitual de gestão do conhecimento científico no contexto acadêmico, tendo por base os processos de comunicação científica.

\section{Palavras-chave}

Gestão do conhecimento. Comunicação científica. Gestão do conhecimento científico. Modelo de gestão do conhecimento científico. Conhecimento científico. Conhecimento científico tácito. Gestão do conhecimento em universidades.

\section{Scientific knowledge management: proposal for a conceptual model based on processes of scientific communication}

\begin{abstract}
Knowledge management has been developed in the context of enterprises and has its research and applications related to the organisational knowledge perspective. Nevertheless, there are other contexts within which knowledge management can be studied, such as the academic context, which is concerned with the scientific knowledge perspective. Whatever is the context in which knowledge management is studied, communication processes constitute a fundamental issue to be taken into account. This piece of research, therefore, aimed to investigate the relationship between knowledge management and the scholarly communication process at a conceptual level, bearing in mind the peculiarities of the scientific knowledge and the academic context. The study is particularly concerned with a theoretical construction of a conceptual model for the knowledge management in the academic context, having the scholarly communication process as its foundation.
\end{abstract}

\section{Keywords}

Knowledge management. Scholarly communication. Scientific knowledge management. Conceptual model of the scientific knowledge management. Tacit scientific knowledge. Knowledge management within the university environment.

\section{INTRODUÇÃO}

Iniciativas de gestão do conhecimento devem necessariamente considerar as características do ambiente no qual são implementadas. Tais características dizem respeito, principalmente, à natureza do conhecimento - bem como as forças que condicionam a sua criação - a cultura que envolve os indivíduos e o seu comportamento em relação à informação e ao conhecimento. Além disso, devem levar em conta, sobretudo, as peculiaridades dos processos de comunicação próprios do ambiente no qual as iniciativas serão implementadas. Desse modo, é imprescindível observar que comunidades de naturezas distintas requerem modelos de gestão do conhecimento que atentem para as suas especificidades. Portanto, a partir das características do contexto no qual se pretende desenvolver a gestão do conhecimento - organizações empresariais, administração pública, ambiente acadêmico ou outros -, deverão ser delineados modelos de gestão do conhecimento apropriados a cada um deles.

Especificamente em relação ao ambiente acadêmico, corporificado principalmente pelas universidades, parecem ser poucas as iniciativas, os estudos ou os modelos de gestão do conhecimento que, de fato, consideram as suas particularidades. Dentre tais particularidades, destacam-se os processos de comunicação científica, a natureza da produção do conhecimento científico e a estrutura e comportamento de comunidades científicas, além da cultura que envolve o ambiente acadêmico.

Este artigo apresenta resultados de pesquisa teórica que teve por objetivo propor um modelo conceitual de gestão do conhecimento científico considerando o contexto do ambiente acadêmico e suas particularidades. O objetivo foi, portanto, investigar a relação, em nível conceitual, entre a gestão do conhecimento e os processos de comunicação científica, tendo em vista as peculiaridades do contexto acadêmico e do conhecimento científico. 


\section{REFERENCIAL TEÓRICO}

O referencial teórico explicita as relações entre os elementos que, com base em análise crítica da literatura, foram identificados como importantes para a construção do modelo conceitual de gestão do conhecimento científico. Assim, o referencial teórico constitui a lente por meio da qual o problema da pesquisa foi compreendido e investigado. Devido à dificuldade de encontrar estudos sobre gestão do conhecimento que observassem a natureza do ambiente acadêmico, do conhecimento científico e dos processos de comunicação científica, um dos principais esforços da pesquisa foi a sinalização de questões que envolvem a gestão desse tipo particular de conhecimento, bem como as implicações do seu ambiente, das características particulares do conhecimento e da comunicação científica.

Tendo em vista o problema proposto na pesquisa - a representação, por meio de um modelo conceitual, da gestão do conhecimento científico em sua vertente tácita e explícita, no contexto acadêmico -, apresentase, a seguir, de maneira breve, a discussão de conceitos e abordagens pertinentes ao estudo sobre i) comunicação científica, ii) aspectos da informação e do conhecimento científico, iii) contexto/cultura científica e iv) a gestão do conhecimento.

\section{Comunicação científica}

O estudo da comunicação científica envolve amplo leque de tópicos e questões complexas. Muitas dessas questões referem-se aos fatores condicionantes do fluxo da informação e do conhecimento, ao comportamento informacional dos atores e suas interações no seio de comunidades científicas, impacto de tecnologias, dentre outros. A comunicação do conhecimento científico abrange os fenômenos compreendidos entre a fase mais incipiente da pesquisa científica - como a identificação do problema a ser estudado - até o momento em que o conhecimento produzido é internalizado por outros cientistas (GARVEY; GRIFFITH, 1979). Portanto, que pode ser entendida como o conjunto de esforços, facilidades, processos dinâmicos e complexos, consensual e socialmente compartilhados, por meio dos quais o conhecimento científico - em sua vertente tácita e explícita - é criado, compartilhado e utilizado. Tais processos também oferecem meios e condições para a interação social entre membros de comunidades científicas, contribuindo, portanto, para a produção, disseminação e uso do conhecimento e, conseqüentemente, para o avanço da ciência.
Naturalmente, é possível afirmar que há uma relação indissociável entre a produção do conhecimento científico e o processo de comunicação a ela subjacente (GARVEY; GRIFFITH, 1979; ZIMAN, 1981; MEADOWS, 1999). Dessa maneira, no contexto das instituições acadêmicas e das comunidades científicas, a criação do conhecimento não pode prescindir da comunicação científica. É neste sentido que Meadows (1999) ressalta a importância da comunicação para a construção do conhecimento científico, ao salientar que a comunicação encontra-se no próprio coração da ciência, sendo ela tão vital quanto a própria pesquisa. Meadows enfatiza que todo esforço é desperdiçado se não forem divulgados os resultados das pesquisas. Destarte, a comunicação científica constitui parte essencial do processo de criação do conhecimento científico.

A criação do conhecimento científico ocorre essencialmente por meio da pesquisas científicas realizadas por pesquisadores-docentes. Durante a realização desses estudos, o pesquisador, um dos atores do processo de comunicação, faz uso do sistema de comunicação em diversos momentos, uma vez que, à medida que produz conhecimento, ele necessariamente o consome. Logo, no início da criação de um novo conhecimento, o esforço de um pesquisador parte daquilo que foi construído anteriormente por outros pesquisadores. Ou seja, o pesquisador nunca parte do marco inicial, pois, no princípio e durante o processo de criação, recorre à literatura de sua especialidade e aos seus pares, e, ao fim, divulga os resultados de sua pesquisa por meio dos veículos de comunicação apropriados à sua área de conhecimento. Torna-se evidente, portanto, que um pesquisador utiliza os canais de comunicação em todo o ciclo do conhecimento - desde a sua criação até a sua divulgação -, o que nos permite afirmar que existe um complexo sistema de comunicação científica que permeia as comunidades científicas e instituições acadêmicas.

Pesquisadores dedicados ao estudo da comunicação científica geralmente dividem os canais de comunicação científica em formais e informais. Embora, hoje, o desenvolvimento e aplicação de tecnologias no processo de comunicação na ciência tenha tornado o limite entre o formal e o informal cada vez mais nebuloso, essa divisão parece ainda ser pertinente. Sobre a relação dos canais formais e informais do processo de criação, estudos revelam indícios de que a comunicação informal é que mais contribui para o fluxo de informação e conhecimento no mundo acadêmico. Braga (1985) ressalta que a comunicação formal é responsável por apenas $20 \%$ de todas as comunicações no processo de geração do conhecimento. Em contrapartida, as 
comunicações que utilizam os canais informais representam $80 \%$ de toda a comunicação nesse processo. Garvey e Griffith (1979) destacam que, pelas suas características, os meios informais são restritos a uma pequena audiência. Entretanto, proporcionam maior interação entre os membros da comunidade, já que pesquisadores de uma mesma área criam teias de comunicação a partir de encontros periódicos e trocas de informações, constituindo assim os colégios invisíveis. Os colégios invisíveis podem ser entendidos como uma rede de interações informais entre os membros de uma mesma área de atividade científica.

É importante destacar que o uso da Internet e de tecnologias emergentes no contexto da comunicação científica tem proporcionado e ampliado, ao longo do tempo, uma série de novas possibilidades e oportunidades de inovação nesse campo. Lagoze e Van de Sompel (2001) corroboram essa idéia, ao afirmarem que a introdução em grande escala das tecnologias de comunicação e informação gerou demanda para o uso da Web para a disseminação dos resultados de pesquisas. Isto fez surgir modelos alternativos para comunicação científica, tais como repositórios institucionais e temáticos, periódicos de acesso aberto, constituindo hoje o Movimento pelo Acesso Aberto à Informação Científica. É importante dar destaque ao seu caráter revolucionário, visto que, além de questionar, tem causado transformações paradigmáticas com significado econômico, tecnológico, político, cultural e social, ocasionando conseqüências, tais como a quebra do monopólio de grandes editores científicos, a criação de canais alternativos de veiculação de literatura científica validada ou não (repositórios institucionais ou temáticos), o aumento da visibilidade da produção científica e o auto-arquivamento por parte dos próprios pesquisadores. Essas transformações incidem diretamente no modo como os indivíduos em uma comunidade científica criam, compartilham e utilizam o conhecimento.

Por fim, com base em Garvey e Griffith (1979), Crane (1972), Meadows (1999), Costa (1999) e outros, entende-se, portanto, que o sistema de comunicação científica exerce importante papel para a criação, compartilhamento e uso do conhecimento científico. Mesmo diante da estreita e cada vez mais frágil divisão entre meios formais de comunicação, é possível ainda sugerir que os formais são mais aptos a compartilhar conhecimento científico em sua vertente explícita. Em contrapartida, o compartilhamento do conhecimento científico tácito requer necessariamente meios informais de comunicação.

\section{Informação e conhecimento científico}

O conhecimento científico explícito pode ser definido como toda a forma de conhecimento codificado, facilmente estruturável e que tem possibilidade de ser comunicado por sistemas estruturados ou meios formais de comunicação. Compreende, então, todas as formas de literatura científica, avaliadas ou não. $\mathrm{O}$ conhecimento científico tácito, por sua vez, refere-se ao que pode ser entendido como o conhecimento ou habilidade que pode ser passada entre cientistas por contatos pessoais, mas não pode ser exposto ou passado em fórmulas, diagramas, descrições verbais ou instruções para ação (COLLINS, 2001). Neste sentido, é o conhecimento baseado também na informação científica, contudo está relacionado com a experiência e a competência do pesquisador, portanto de difícil sistematização e representação. Diz respeito àquele conhecimento que é mais bem transferido e assimilado informalmente. Somente parte do conhecimento científico tácito é possível ser formalizada. Quando há o compartilhamento do conhecimento científico tácito, há necessariamente a utilização da comunicação informal. Por esta razão, talvez, é atribuído à comunicação científica informal grande importância na produção do conhecimento científico. A relação entre conhecimento científico tácito e comunicação informal é explorada com maior profundidade em Leite (2006).

A partir da interação entre conhecimento científico explícito - o conhecimento científico registrado, a literatura científica - e o conhecimento científico tácito - aquilo que os pesquisadores sabem, aprenderam e é comunicado por meios impessoais, e não estruturados -, torna-se viável a criação de um novo conhecimento científico. A díade conhecimento tácito e explícito nos remete inicialmente a Michael Polanyi (1966), referindose inicialmente ao próprio conhecimento científico. Entretanto, a teoria de criação do conhecimento a partir das duas dimensões diz respeito ao modelo construído por Nonaka e Takeuchi (1997), responsáveis pela ampla disseminação dos conceitos de conhecimento tácito e explícito, por meio da abordagem de gestão do conhecimento.

\section{Comunidades científicas e acadêmicas}

É importante ressaltar que universidades, como comunidades acadêmicas, constituem elementos do sistema científico. São consideradas ainda como o cerne da produção do conhecimento, e os processos de comunicação científica permeiam boa parte de suas atividades, o que permite tanto as trocas internas de 
conhecimento quanto externas, em interação com comunidades científicas. As comunidades científicas, por sua vez, podem ser entendidas como o agrupamento de pares que compartilham um tópico de estudo, desenvolvem pesquisas e dominam um campo de conhecimento específico, em nível internacional (COSTA, 1999). Tais comunidades, de caráter disciplinar, influenciam fortemente os processos de comunicação científica em uma universidade. Como as atividades de ensino e pesquisa realizadas por pesquisadores-docentes de uma universidade são comumente organizadas em áreas do conhecimento, tem-se que, por conseqüência, comunidades científicas de diferentes áreas do conhecimento estão representadas em uma universidade. Assim, os processos de comunicação científica e a própria produção do conhecimento científico, bem como a cultura de uma universidade, são diretamente influenciados por diferentes comunidades científicas.

\section{Contexto e cultura científica}

Tanto os processos de comunicação científica, quanto o próprio conhecimento científico - e mesmo a gestão do conhecimento - estão necessariamente relacionados e envolvidos por um determinado contexto, no caso, o ambiente acadêmico e a cultura do meio científico. $\bigcirc$ ambiente científico possui características culturais próprias que o difere de outros contextos, por exemplo, o das empresas, comunitário ou governamental. Essas características culturais estão relacionadas aos valores, pressupostos e crenças que são partilhados entre os indivíduos que vivem no ambiente e são reafirmados cotidianamente por estarem embrenhados em suas atividades e relações sociais. Especificamente, a cultura e o contexto da ciência e do conhecimento científico moldam as dinâmicas das interações dentro das comunidades, sejam elas científicas ou acadêmicas, e legitimam comportamentos, práticas e processos. Assim, tanto os processos relacionados à criação do conhecimento científico, quanto os processos voltados para a sua comunicação, por exemplo, são moldados e adequados à cultura proveniente do ambiente científico. Sobre essa questão, Meadows (1999, p. 245) destaca que pesquisadores trabalham muitas vezes de modo inconsciente, com base nas práticas instituídas da comunidade científica, as quais, por sua vez, são determinadas por sua história e normas sociais. Por esta razão, a gestão do conhecimento científico deve também levar em consideração as características culturais próprias desse ambiente.
No contexto da ciência, pode-se mencionar que os pressupostos da cientificidade, bem como aspectos da visão metorniana da ciência, as concepções do campo científico de Bourdieu e aspectos do novo modo de produção do conhecimento de Gibbons, por exemplo, constituem modelos que contribuem para o entendimento da formação de uma cultura científica. Essa cultura científica, no nível das instituições acadêmicas - especialmente as universidades -, certamente é refletida na cultura da organização universitária. Assim, a cultura organizacional, entendida com base no conceito de Schein e Callaghan (2001, p. 10), relaciona-se ao padrão de pressupostos básicos partilhados e aprendidos por membros de uma comunidade à medida que é capaz de solucionar seus problemas, que têm funcionado bem o suficiente para serem considerados como válidos. Por essa razão, segundo o autor, são ensinados aos novos membros como sendo o modo correto de perceber, pensar e sentir em relação àqueles problemas. Isso significa dizer, em outras palavras, que a cultura organizacional de uma universidade reflete aspectos de uma cultura científica - os quais são partilhados entre os membros das comunidades científicas -, visto que está inserida em um sistema científico maior. Essa cultura científica/organizacional regula as interações, influencia a percepção e contribui para a formação dos valores próprios de uma instituição acadêmica.

\section{Gestão do conhecimento}

A gestão do conhecimento é um processo complexo e intimamente relacionado com processo de comunicação nas organizações (SMOLIAR, 2003; IVES et al., 1998; THEUNISSEN, 2004). As duas abordagens - gestão do conhecimento e processos de comunicação - possuem princípios compatíveis e objetivos convergentes em diversos momentos, sobretudo durante a fase de compartilhamento ou disseminação de conhecimento. A gestão do conhecimento, de forma abrangente, referese ao planejamento e controle de ações (políticas, mecanismos, ferramentas, estratégias e outros) que governam o fluxo do conhecimento, em sua vertente explícita - e para isso englobam práticas da gestão da informação - e sua vertente tácita. $\bigcirc$ planejamento e controle de ações pressupõem a identificação, aquisição, armazenagem, compartilhamento, criação e uso do conhecimento tácito e explícito, com o fim de maximizar os processos organizacionais em qualquer contexto. Todo esse processo viabiliza-se mediante o substrato comunicacional. 
É importante destacar o entendimento dos autores sobre a relação entre a gestão do conhecimento e a gestão da informação. Com base na definição anterior, entendese que a gestão do conhecimento engloba as práticas e metodologias de gestão da informação quando diz respeito principalmente aos processos relacionados à captura, armazenamento, recuperação de uma parcela do conhecimento tácito, o qual neste momento é reduzido à estruturas de informação. Muito embora isso aconteça, a gestão do conhecimento não pode ser reduzida à gestão da informação, nem tampouco confundida, mesmo que ela se aproprie desta. No momento em que, por exemplo, o tipo de compartilhamento do conhecimento exige que ele seja veiculado por meio de sistemas formais (que permitem o seu 'armazenamento e recuperação'), o conhecimento é reduzido a estruturas de informação. Esta, por sua vez, poderá vir a disparar ou desencadear o processo de geração do conhecimento no indivíduo.

A maioria dos autores estudiosos da ciência da informação considera que a área deve se ocupar do que Le Coadic (2004), entre outros autores, chama de conhecimento registrado. Críticos da gestão do conhecimento, portanto, costumam considerar que o conhecimento, uma vez registrado, é informação. Bouthillier e Shearer (2002), no entanto, consideram o argumento de que conhecimento registrado (ou explícito) é informação como uma "avaliação inadequada das dimensões qualitativas dos vários tipos de informação e de conhecimento criados, usados e transferidos".

Sobre os estudos da gestão do conhecimento na ciência da informação, vale a pena retomar as abordagens de Belkin (1978) e Brookes (1980) para a informação e a ciência da informação, respectivamente. No trabalho de Belkin, o conceito de informação é expresso tanto em nível cognitivo, quanto em nível lingüístico, assim representado (BELKIN, 1978, p. 81):

\section{TEXTOS}

GERADORES
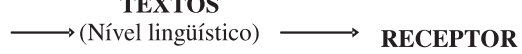

\section{INFORMAÇÃO $\longleftrightarrow$} (Nível cognitivo)

ESTADO ESTADO DE
CONHECIMENTO

No nível lingüístico, o autor insere a informação em um processo de comunicação entre seres humanos. No nível cognitivo, a abordagem de Belkin centra-se nas transformações ocorridas nos estados de conhecimento do indivíduo, quando este recebe a informação capaz de preencher uma necessidade ou lacuna. Um esforço maior

permite ver, nos dois níveis em que é expresso, o conceito de informação de Belkin, a comunicação como parte da gestão.

A abordagem de Brookes (1980) e a análise que o autor faz sobre os campos de atuação da ciência da informação e - como se percebe atualmente - da gestão do conhecimento complementam a abordagem de Belkin. Batista et al. (2007) estudaram essas questões, destacando que Brookes

considera que, embora a coleta, a organização e o acesso às fontes de informação sejam essenciais para a prática profissional nas bibliotecas, o foco da prática profissional deveria ser a transformação da informação em conhecimento pessoal (grifo dos autores). Por isso, argumenta que o mundo da ciência da informação deve ser visto como um mundo mais amplo e distinto do mundo da documentação e biblioteconomia.

Esse mundo mais amplo e distinto, na visão de Brookes, está situado nas interações entre os Mundos 2 e 3 de Popper (1975). Como é possível notar, Brookes, com base na abordagem dos Três Mundos de Popper, propõe uma relação entre informação e conhecimento nos estudos da ciência da informação baseada, principalmente, na noção de "espaços mentais únicos". O autor chama a atenção para o fato de que os eventos do "Mundo 2" individualidades mentais - ocorrem em espaços individuais privados (grifo do autor), sendo, portanto, subjetivos. Para objetivá-los, é necessário expressá-los e depositar os registros no Mundo 3, onde são acessíveis a outros e podem, portanto, ser criticamente considerados.

Brookes explora o Mundo 3 de Popper como o da biblioteconomia e da ciência da informação, ressaltando, entretanto, que o trabalho prático (grifo do autor) dos profissionais da área é coletar e organizar para uso os registros do Mundo 3. Suas atribuições teóricas (idem) são o estudo das interações entre o Mundo 2 e o Mundo 3, "para os descrever e explicar, se puderem, e, então, ajudar na organização do conhecimento (idem), mais do que de documentos (idem), para o uso (grifo nosso) mais efetivo" (BROOKES, 1980, p. 128).

Uma observação importante de Brookes para os estudos da gestão do conhecimento na ciência da informação, a partir das abordagens dos três mundos de Popper, é que:

essa abordagem, portanto, permite-nos escapar das subjetividades da velha abordagem de 2000 anos para as teorias do conhecimento e da psicologia subjetiva, 
assim como da filosofia tradicional. Mais que isso, ao adotar a interação entre os Mundos 2 e 3 como nosso campo de estudos, nós estamos reivindicando a propriedade de uma disciplina que nenhuma outra disciplina já reivindicou.

O argumento de Brookes, como pode ser notado e está explícito em seu texto, é que a ciência da informação não deveria ser uma combinação de abordagens de várias disciplinas, como a lingüística, ciência da computação, entre outras. Isso porque a área tem, na verdade, segundo o autor, seu próprio território, seus próprios problemas e sua visão própria dos problemas humanos.

Observe-se que o que Brookes chama de "espaços mentais únicos" pode perfeitamente ser considerado como o que Nonaka e Takeuchi (1997) chamam de "conhecimento tácito". Assim, o que Brookes chama de "objetivação" dos espaços individuais fornece a base para os processos de conversão do conhecimento de Nonaka e Takeuchi, mais especificamente os processos de socialização, internalização e externalização. Conforme concluem Batista et al. (2007),

a análise do modelo de Nonaka e Takeuchi [...] mostra que é a gestão do conhecimento, que, ao se preocupar em descrever e analisar os processos de conversão das duas formas de conhecimento (tácito ou subjetivo e explícito ou objetivo), realiza a segunda proposta feita por Brookes aos cientistas da informação, isto é, estudar as interações entre os Mundos 2 e 3 de Popper.

São vários os modelos identificados na literatura para o estudo da gestão do conhecimento. A análise desses vários modelos ao longo do estudo fundamentou a consideração de que a gestão do conhecimento engloba práticas da gestão da informação, muito embora não possa ser confundida nem tampouco reduzida a ela. Assim, a gestão da informação é aplicável, principalmente, aos processos relacionados à captura, armazenamento e recuperação de apenas uma parcela do conhecimento tácito, o qual, neste momento, é necessariamente reduzido a estruturas de informação. Desse modo, a gestão do conhecimento possui intenções diferenciadas que se sobrepõem à gestão da informação, uma vez que se volta, também, para criação e agregação de valor ao conhecimento. A gestão da informação, por seu turno, lida com a parcela do conhecimento tácito que foi explicitado e passível de ser comunicado por meio de sistemas formais de comunicação e, sendo assim, tornase um dos meios necessários para o alcance das pretensões da gestão do conhecimento.
Entretanto, quando se tem em questão o conhecimento tácito de fato, a gestão do conhecimento é responsável pela condução dos processos relacionados ao fluxo e apropriação do conhecimento. Este, por seu turno, não pode ser manipulado, no sentido estrito da palavra. A 'intencionalidade maior' refere-se, em última análise, em fazer com que todo o tipo de conhecimento seja valorizado, compartilhado, utilizado, retido na organização, com o fim de que os indivíduos criem conhecimento, aprendam mais e da melhor forma. Está preocupada com a criação de condições férteis, a condução de situações ótimas e viabilizadoras para que o conhecimento seja criado, compartilhado, assimilado e convertido em benefícios aplicáveis à consecução dos objetivos de uma determinada organização. Todas esses aspectos pressupõem elementos a mais do que a gestão da informação sozinha pode oferecer. Os argumentos apresentados que ilustram a relação entre a gestão da informação e do conhecimento, resumidos, em última instância, na interação entre conhecimento tácito (subjetivo) e conhecimento explícito (objetivo), podem ser graficamente representados pela figura 1. Portanto, a gestão da informação é um dos componentes da gestão do conhecimento.

A literatura sobre a gestão do conhecimento é fortemente influenciada pela teoria de criação do conhecimento organizacional cunhada por Nonaka e Takeuchi (1997), e, embora se deva levar em consideração o contexto cultural específico do desenvolvimento da teoria dos autores japoneses - organizações empresariais -, ainda sim o seu modelo é uma contribuição sólida ao corpo de conhecimento que fundamenta a gestão do conhecimento. Resumidamente, a construção teórica dos autores é apresentada sob a ótica de duas dimensões. A primeira, ontológica, tem em vista o conhecimento organizacional em oposição à criação do conhecimento

\section{FIGURA 1 \\ Relação entre gestão do conhecimento e gestão da informação}

Gestão do Conhecimento

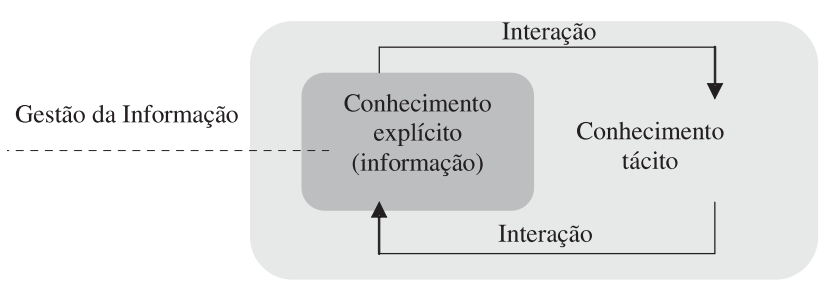

Fonte: adaptado de Leite (2006) 
individual, é relacionada aos níveis de entidades criadoras do conhecimento (individual, grupal, organizacional e interorganizacional). Parte do entendimento de que o conhecimento só é criado por indivíduos, ou seja, uma organização não pode criar conhecimento por si só, é um processo que amplia organizacionalmente o conhecimento criado pelos indivíduos. A segunda, a dimensão epistemológica, tem como base fundamental a distinção entre conhecimento tácito e explícito, onde o conhecimento que pode ser expresso em palavras e números (explícito), representando apenas a ponta do iceberg do conjunto de conhecimentos como um todo. A partir dessa sistematização, ocorre a espiral da criação do conhecimento, que surge no momento em que "a interação entre conhecimento tácito e conhecimento explícito eleva-se dinamicamente de um nível ontológico inferior até níveis mais altos" (NONAKA; TAKEUCHI, 1997, p. 62). A explicação da espiral de criação do conhecimento organizacional constitui o âmago da teoria dos autores, em que, por meio dos quatro modos de conversão (socialização, externalização, combinação e internalização), que ocorrem a partir da interação entre conhecimento tácito e explícito, é construído um novo conhecimento (NONAKA; TAKEUCHI, 1997, p. 62). A concepção do conhecimento tácito e explícito, em uma dinâmica e contínua interação que torna possível a criação de novo conhecimento, é a premissa básica da teoria. Os autores ressaltam que o processo de criação do conhecimento é fruto de uma interação constante e dinâmica entre os dois tipos de conhecimento. Tal interação é moldada pelas mudanças entre os diferentes modos de conversão, e estes, por seu turno, sofrem influência de vários outros fatores. $\mathrm{Na}$ sua visão, conhecimento explícito e conhecimento tácito são entidades complementares e não excludentes, interagem um com o outro e realizam trocas nas atividades criativas desempenhadas pelos seres humanos. Os autores acrescentam ainda que o modelo dinâmico de criação do conhecimento é fundamentado no pressuposto de que o conhecimento humano é criado e expandido mediante a interação social entre o conhecimento tácito e o conhecimento explícito. A essa interação, dão o nome de espiral do conhecimento.

A teoria embutida no referencial teórico sugere, em um primeiro momento, que a comunicação científica é crucial para a gestão do conhecimento científico. Quer se dizer com isso que, de acordo com o referencial desenvolvido, em nível conceitual, a comunicação científica é o substrato fundamental para o desenvolvimento da gestão do conhecimento científico no contexto acadêmico. Atrelado a isso, os pressupostos da gestão do conhecimento, os aspectos relacionados ao conhecimento e, mais especificamente, ao conhecimento científico, juntamente com processos de comunicação científica, são elementos que influenciam sistemicamente o desenvolvimento da gestão do conhecimento científico no contexto acadêmico. Da mesma forma, as questões relacionadas com o conhecimento e os pressupostos da gestão do conhecimento influenciam e são influenciados pela comunicação científica. Todos esses elementos estão dispostos em um contexto acadêmico, o qual possui características culturais próprias que são influenciadas por uma cultura científica maior. O contexto acadêmico, por sua vez, influencia todos os relacionamentos entre comunicação científica, aspectos do conhecimento e pressupostos da gestão do conhecimento, refletindo, por conseqüência, na gestão do conhecimento científico. Outra relação que se quer destacar está relacionada ao argumento de que as atividades de ensino e pesquisa realizadas por pesquisadores-docentes de uma universidade estão organizadas em diferentes áreas do saber. Por esta razão e conseqüentemente, as comunidades científicas de diferentes áreas do conhecimento influenciam os processos de comunicação científica, a própria produção do conhecimento científico, assim como a cultura científica/organizacional de uma universidade. Esses seriam os elementos e relacionamentos essenciais, em nível conceitual, de um sistema de gestão do conhecimento científico.

O referencial teórico, criado a partir da discussão dos conceitos de conhecimento científico, comunicação científica, gestão do conhecimento, cultura científica/ organizacional e suas inter-relações, norteou a construção do modelo teórico de gestão do conhecimento científico em sua vertente tácita e explícita, tendo por base os processos de comunicação científica. Esta, por seu turno, está relacionada com o planejamento e controle de ações (políticas, mecanismos, ferramentas, estratégias e outros) que governam o fluxo do conhecimento científico em sua vertente tácita e explícita e tem como substratos os processos de comunicação científica com o fim de apoiar e maximizar a geração de novos conhecimentos e o ensino.

\section{METODOLOGIA}

A metodologia empregada no estudo envolveu diferentes momentos. Inicialmente, foi construído, com base na análise crítica da literatura e extração de conceitos, o referencial teórico constituindo o modelo da pesquisa. Este ofereceu os fundamentos a partir dos quais o 
problema de pesquisa foi observado e discutido. Em seguida, norteados pelo referencial teórico, foram determinados a abordagem metodológica e os procedimentos metodológicos propriamente ditos, cujo detalhamento é feito a seguir.

Por se tratar de tema ainda pouco explorado - a convergência de abordagens da comunicação científica e da gestão do conhecimento -, existe pouco conhecimento a respeito. Dessa maneira, o esforço da pesquisa foi direcionado para a construção teórica de uma base que fundamentasse um modelo conceitual de gestão do conhecimento científico, com aporte na literatura de cada uma das abordagens.

modelo conceitual de gestão do conhecimento científico proposto foi, portanto, delineado a partir da construção teórica realizada a partir da literatura e de entrevistas realizadas com pesquisadores-docentes de diferentes áreas do conhecimento. A literatura estudada circunscreve principalmente os campos da ciência da informação e da administração. Os textos selecionados são trabalhos científicos relevantes (artigos, teses, dissertações e livros) dos tópicos relacionados à comunicação científica, conhecimento científico e gestão do conhecimento cujas temáticas incidem, direta ou indiretamente, sobre o tema proposto, norteando os elementos do referencial teórico desenvolvido para o estudo. $O$ período de realização das buscas em bases de dados foi compreendido entre julho de 2004 a dezembro de 2005, e não houve restrição de ano das publicações* . Os documentos recuperados por meio das buscas foram classificados em três amplas categorias: 1) aspectos conceituais da informação e conhecimento; 2) comunicação científica; 3) gestão do conhecimento.

No que diz respeito às entrevistas com pesquisadoresdocentes de diferentes áreas do conhecimento, foram adotados os estágios sugeridos por Kvale (1996), nomeadamente, tematização, planejamento ou delineamento, entrevista, transcrição, análise, apresentação. $\mathrm{O}$ roteiro utilizado no instrumento de coleta de dados foi planejado tendo por base conceitos e teorias que constituem parte do referencial teórico desenvolvido para o norteamento do estudo, como sugerem Bauer e Gaskell (2002, p. 66). Os autores orientam que, na preparação e planejamento do instrumento de coleta de dados, é necessário que o pesquisador já tenha desenvolvido um referencial teórico ou conceitual que guie sua investigação e que tenha

\footnotetext{
* Os procedimentos de busca e as principais fontes de informação encontram-se descritos em Leite (2006).
}

identificado os conceitos centrais e os temas que deverão ser vistos na pesquisa. De acordo com isto, o roteiro da entrevista foi fundamentado na combinação de uma análise crítica da literatura pertinente, no reconhecimento do campo - segundo os autores, isto pode incluir observações e/ou algumas conversações preliminares com algumas pessoas relevantes -, discussões com colegas experientes e algum pensamento criativo. Os pesquisadores-docentes sujeitos da pesquisa, vinculados à Universidade de Brasília, foram selecionados com base nos seguintes critérios: ser líder de grupo de pesquisa produtivo; ter produção científica recente; orientar alunos de mestrado e doutorado; ter envolvimento com o ensino em nível de graduação e pós-graduação. A seleção desses sujeitos justifica-se por serem atores que estão diretamente relacionados com a produção e difusão do conhecimento científico. Todas as informações para a seleção do universo foram coletadas na Plataforma Lattes do Conselho Nacional de Ciência e Tecnologia (CNPq). Após o preenchimento dos critérios, 18 pesquisadores foram contatados formalmente via correio eletrônico. Desse total, 15 retornaram o contato e dispuseram-se ser entrevistados. Três deles constituíram o universo do pré-teste. $\mathrm{Na}$ constituição do universo da pesquisa, as áreas do conhecimento foram assim representadas: ciências engenharia (física, química, biologia, engenharia mecânica); ciências sociais e humanas (sociologia, psicologia, educação); humanidades (educação, história, lingüística).

\section{GESTÃO DO CONHECIMENTO CIENTÍFICO: UM MODELO CONCEITUAL}

A gestão do conhecimento científico no contexto acadêmico pode ser estudada a partir de duas perspectivas distintas. A primeira perspectiva, a vertical, diz respeito ao âmbito das comunidades científicas. Nesse ângulo de análise, as comunidades científicas, como mencionadas anteriormente, são entendidas como o agrupamento de pares que compartilham um tópico de estudo, desenvolvem pesquisas e dominam um campo de conhecimento específico, em nível internacional (COSTA, 1999). A segunda perspectiva, a horizontal, está relacionada às instituições de ensino e pesquisa. Neste ângulo, adota-se o conceito de comunidades acadêmicas.

A perspectiva da gestão do conhecimento científico no contexto das comunidades científicas, no plano vertical, está relacionada com a gestão do conhecimento produzido por uma disciplina, tópico ou campo específico 
do saber. Logo, a gestão do conhecimento científico no âmbito de comunidades científicas não possui caráter institucional, e sim disciplinar. Nesse sentido, a caracterização do conceito de comunidade científica como o conjunto de pesquisadores que compartilham interesse sobre tópicos ou áreas específicas, em nível internacional, vem ao encontro daquilo que caracteriza as redes. Probst et al. (2002, p. 82) afirmam que uma rede é caracterizada por um interesse básico comum entre seus membros, além de uma orientação pessoal e participação voluntária, e suas relações estão baseadas no princípio das trocas. As relações de compartilhamento e os fluxos de informação e conhecimento no seio das comunidades científicas ocorrem por meio dos colégios invisíveis. Diferentemente das comunidades acadêmicas, as comunidades científicas não possuem fronteiras nem características organizacionais.

Entretanto, a perspectiva da gestão do conhecimento científico voltada às instituições de ensino e pesquisa o plano horizontal, neste estudo, uma universidade está relacionada com o conceito de comunidades acadêmicas. Comunidades acadêmicas dizem respeito ao agrupamento de membros de uma instituição acadêmica envolvidos com atividades de ensino e pesquisa, constituindo os seus recursos humanos para a pesquisa, compartilhando ou não interesses comuns em seus tópicos de estudo. Porém, seus pesquisadores pertencem, individualmente, a grupos de interesse em tópicos específicos sem limites geográficos, denominados anteriormente comunidades científicas. Nessas comunidades científicas, há constante e intenso compartilhamento de conhecimento, constituindo, no tópico de interesse específico do pesquisador, o principal locus de comunicação e troca de conhecimentos.

Percebe-se que as duas perspectivas para se abordar a gestão do conhecimento científico estão interrelacionadas. Embora seja possível e necessário definir o ângulo de análise, é importante destacar que não é possível uma abordagem excluir a outra. A razão disso está em dois argumentos: i) membros de comunidades científicas, de uma maneira geral, possuem vínculo com instituições de ensino e pesquisa; ii) pesquisadoresmembros das comunidades acadêmicas pertencem individualmente à comunidades científicas específicas. Assim, a produção científica de uma instituição de ensino e pesquisa, representada pelo conjunto da produção científica dos pesquisadores que mantêm vínculo institucional com ela, constitui, ao mesmo tempo, a produção científica de diferentes comunidades científicas. Por exemplo, a comunidade científica que se dedica ao estudo das publicações eletrônicas possui uma quantidade $\mathrm{X}$ de membros. Toda a produção científica desses membros constitui o total de conhecimento produzido por esta comunidade. Porém, ao mesmo tempo, cada um dos membros da comunidade científica de publicações eletrônicas possui vínculo com a alguma instituição de ensino e/ou pesquisa e, individualmente, contribui para a produção científica total da sua instituição - comunidade acadêmica. $\bigcirc$ que se quer destacar é que os dois níveis de análise se sobrepõem. Assim sendo, várias comunidades científicas podem 'cortar' transversalmente determinada comunidade acadêmica. Esta relação pode ser visualizada na figura 2.

\section{FIGURA 2}

\section{Comunidade acadêmica e comunidades científicas}

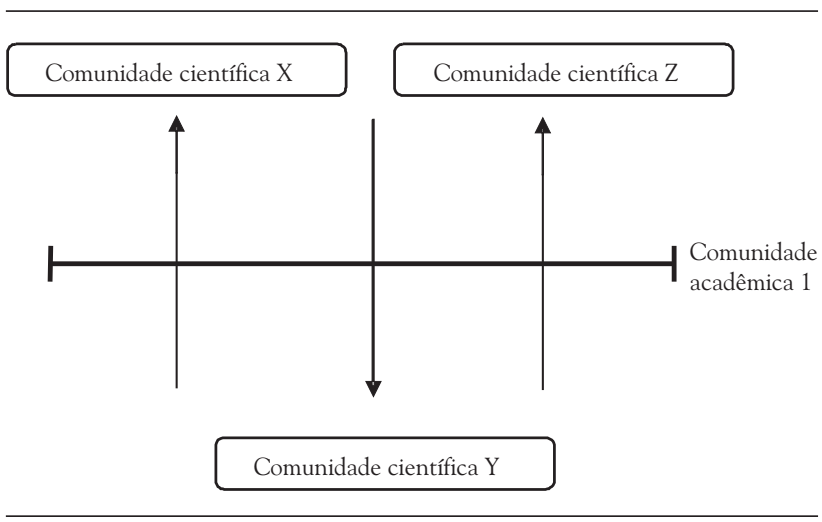

Nesse estudo, a perspectiva adotada é a gestão do conhecimento científico em comunidades acadêmicas, no caso, uma universidade. As implicações das comunidades científicas para esse processo serão exploradas quando da apresentação do modelo conceitual de gestão do conhecimento científico.

Ademais, concorda-se com Wilson (1999, p. 11), ao conceber um modelo como interpretação explícita do entendimento de determinada situação, ou simplesmente de idéias a respeito da situação. Segundo o autor, um modelo pode ser expresso por fórmulas matemáticas, símbolos ou palavras, embora seja essencialmente a descrição de entidades, processos ou atributos e as relações entre eles. Pode ser prescritivo ou ilustrativo, mas, acima de tudo, deve ser útil. Wilson põe em relevo a importância de modelos conceituais no estudo de sistemas organizacionais. De acordo com o autor, esses modelos são utilizados com o fim de clarear determinada área de estudo, ilustrar um conceito, determinar a estrutura e a lógica de uma situação e ser útil como prérequisito para o desenho de um sistema ou projeto. 
modelo conceitual de gestão do conhecimento científico, ilustrado na figura 3 , bem como os relacionamentos entre seus elementos, é derivado dos seguintes procedimentos: análise da literatura; criação do referencial teórico a partir da análise e discussão da literatura; construção teórica sobre a relação entre gestão do conhecimento e processo de comunicação científica, análise e discussão das entrevistas realizadas com pesquisadores de diferentes áreas do conhecimento. Os resultados obtidos com o alcance dos objetivos específicos da pesquisa (LEITE, 2006) fundamentaram, naturalmente, a construção do modelo conceitual de gestão do conhecimento. Nesse artigo, contudo, é ressaltado apenas o delineamento do modelo.

A versão textual do modelo conceitual de gestão do conhecimento científico encontra-se estruturada na seguinte ordem:

1. descrição dos elementos macro do modelo e suas relações (comunidades científicas, comunidades acadêmicas, comunicação científica, cultura científica/organizacional e gestão do conhecimento);

2. descrição dos processos constituintes da gestão do conhecimento;
3. descrição e detalhamento dos relacionamentos entre os elementos macro e os processos de gestão do conhecimento.

\section{Descrição dos elementos macro do modelo e suas relações}

Como se pode observar, os principais elementos que constituem o modelo conceitual resultante deste estudo são as comunidades científicas, a comunidade acadêmica, a comunicação científica, a cultura científica e organizacional e a gestão do conhecimento. Cada um desses elementos e suas relações são descritos a seguir.

1.1. Comunidades científicas: o modelo proposto adota a definição traçada por Costa (1999), em que comunidades científicas podem ser entendidas como o agrupamento de pares que compartilham um tópico de estudo, desenvolvem pesquisas e dominam um campo de conhecimento específico, em nível internacional.

1.2. Comunidade acadêmica: diz respeito ao agrupamento de membros de uma instituição acadêmica envolvidos com atividades de ensino e pesquisa, constituindo os seus recursos humanos para a pesquisa, compartilhando ou não interesses comuns em seus tópicos de estudo. No entanto, pertencem individualmente a grupos de interesse em tópicos específicos, sem limites geográficos, denominados comunidades científicas.

\section{FIGURA 3}

Modelo conceitual de gestão do conhecimento científico no contexto acadêmico

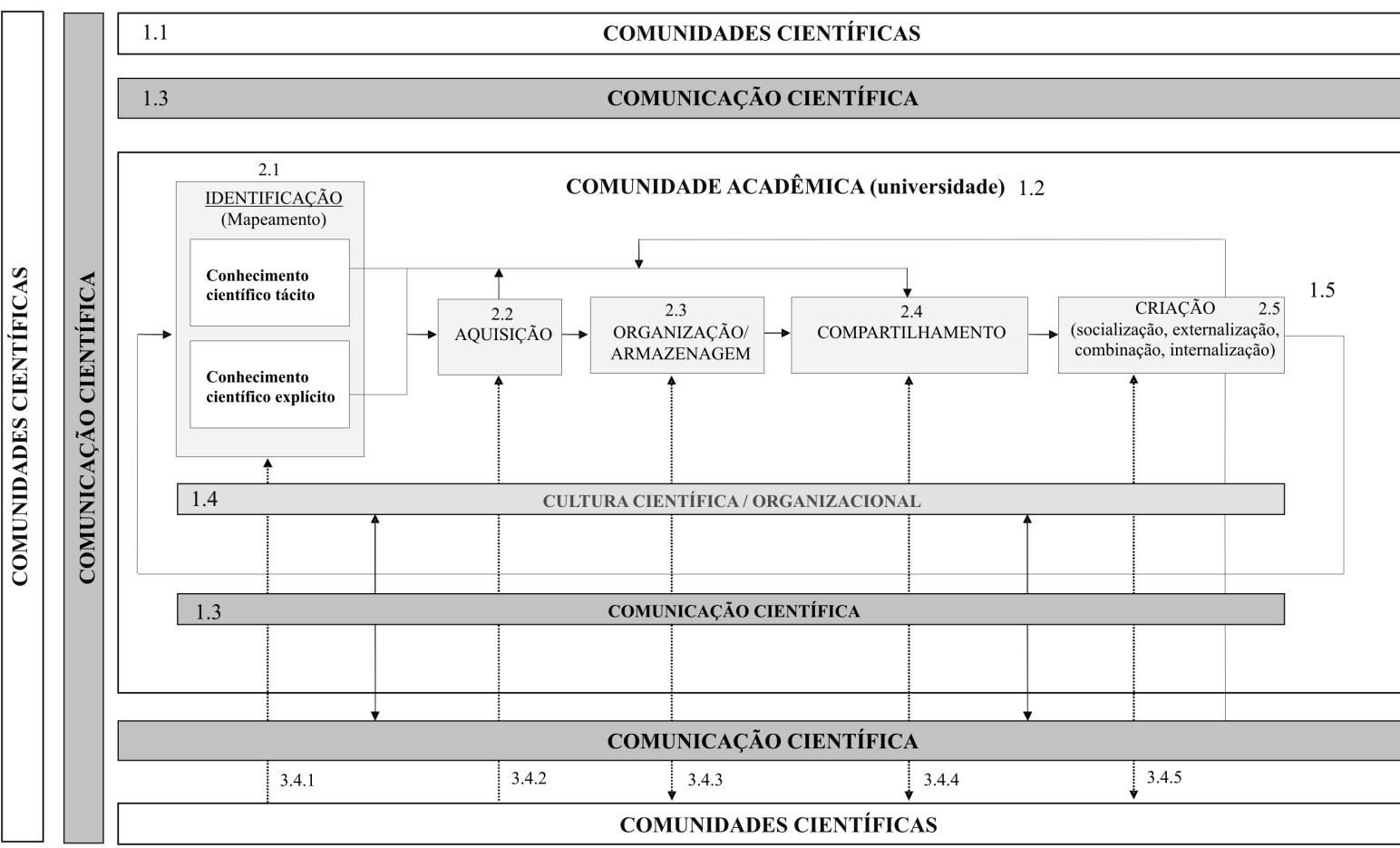


1.3. Comunicação científica: o conjunto de esforços, facilidades e processos dinâmicos e complexos, consensual e socialmente compartilhados, a partir dos quais o conhecimento científico, em sua vertente tácita e explícita, é criado, compartilhado e utilizado. Tais processos oferecem, também, meios e condições para a interação social entre membros de comunidades científicas, contribuindo, portanto, para a produção, disseminação e uso do conhecimento, e, conseqüentemente, o avanço da ciência.

1.4. Cultura científica/organizacional: a cultura científica está relacionada a normas, valores, forças, costumes e pressuposições consensual e socialmente compartilhados em comunidades científicas em seu sentido mais amplo - pesquisadores de todas as áreas do conhecimento - que influenciam e orientam a dinâmica das interações entre eles e a prática científica. Por conseqüência, necessariamente condicionam a produção do conhecimento científico. A cultura organizacional, por sua vez, segundo o conceito de Schein e Callaghan (2001, p. 10) diz respeito ao padrão de pressuposições básicas partilhadas e aprendidas por um grupo à medida que foram capazes de solucionar seus problemas de adaptação externa e de integração interna e, por essa razão, ensinadas aos novos membros como sendo o modo correto de perceber, pensar e sentir em relação àqueles problemas. No modelo proposto, a definição de cultura científica e organizacional requer, obviamente, sobreposição dos dois conceitos. No contexto de uma comunidade acadêmica, uma universidade, a cultura organizacional certamente reflete elementos da cultura científica.

1.5. Gestão do conhecimento: refere-se ao planejamento e controle de ações (políticas, mecanismos, ferramentas, estratégias e outros) que governam o fluxo do conhecimento, em sua vertente explícita - engloba práticas da gestão da informação - e em sua vertente tácita. O planejamento e controle de ações pressupõem a identificação, aquisição, armazenagem, compartilhamento, criação e uso do conhecimento tácito e explícito, com o fim de maximizar os processos organizacionais em qualquer contexto. Todo esse processo viabiliza-se mediante o substrato comunicacional.

\section{Relação entre os principais conceitos do modelo}

A relação conceitual entre os principais elementos do modelo fundamenta-se no pressuposto de que a gestão do conhecimento em comunidades acadêmicas, no caso uma universidade, tem como substrato os processos de comunicação científica e a cultura científica/ organizacional. A comunicação científica é subjacente à interação dos membros da comunidade acadêmica em suas respectivas comunidades científicas e, internamente, em sua comunidade acadêmica. A cultura científica/organizacional, por sua vez, influencia e é influenciada pelos processos de comunicação científica. Além disso, é a cultura, tanto científica quanto organizacional, que legitima os meios de comunicação. Da mesma forma, são os processos de comunicação que veiculam os valores e crenças que influenciam o desenvolvimento de uma cultura, conforme evidenciado nas entrevistas com pesquisadores. Em contrapartida, a partir dos processos de comunicação científica, a comunidade acadêmica, por meio de seus pesquisadores, interage com as comunidades científicas, e estas servem de input de conhecimentos para o pesquisador individualmente e para ela própria.

\section{Descrição dos processos constituintes da gestão do conhecimento científico}

2.1. Identificação: refere-se ao processo de mapeamento do conhecimento da comunidade acadêmica conhecimentos internos à instituição - em sua vertente tácita e explícita e também ao mapeamento do conhecimento externo à instituição proveniente de comunidades científicas. Dessa forma, o objetivo do mapeamento do conhecimento no ambiente acadêmico é responder quem pesquisa o que e onde. Trata-se do mapeamento das fontes de informação, da produção científica, das competências e habilidades científicas internas à instituição e das fontes de informação e competências científicas críticas e externas, as quais, possivelmente, estão relacionadas às atividades científicas da instituição. Este elemento foi derivado tanto da literatura quanto das entrevistas, nas quais os respondentes expressaram percepções que direta ou indiretamente embutem o conceito.

2.2. Aquisição: está relacionada com o processo de aquisição de conhecimentos internos e externos mapeados anteriormente - que são necessários para proporcionar a criação e manutenção de conhecimentos e competências científicas da comunidade acadêmica. A aquisição do conhecimento científico em sua vertente explícita e a externalização de parte do conhecimento científico tácito permitem, em um processo imediatamente posterior, que ele seja armazenado. A aquisição do conhecimento científico em sua vertente tácita, não explicitável, não torna possível a sua armazenagem; essa forma de conhecimento é adquirida 
e, com base no modelo conceitual de gestão do conhecimento científico, é diretamente compartilhada, não sendo possível seu armazenamento e recuperação.

2.3. Armazenagem/organização: processo relacionado com a organização e armazenagem do conhecimento científico explícito com o objetivo de torná-lo facilmente recuperável. No modelo conceitual de gestão do conhecimento científico, os itens de conhecimento registrado são organizados e armazenados conforme os padrões utilizados na Iniciativa dos Arquivos Abertos e do Acesso Livre.

2.4. Compartilhamento: processo que tem como pressuposto básico e primordial para a transformação de informações e experiências isoladas em algo que toda a organização possa utilizar (PROBST et al., 2002). Após o conhecimento ter sido mapeado, adquirido e organizado/ armazenado, de acordo com o modelo proposto, ele deve ser compartilhado. O compartilhamento do conhecimento científico explícito ocorre pelos meios de comunicação formais, enquanto que o compartilhamento do conhecimento científico tácito se dá por meios informais, conforme os aspectos ressaltados ao longo das entrevistas realizadas.

2.5. Criação: o processo de criação constitui um dos elementos essenciais da gestão do conhecimento. Está relacionado com a criação de novas habilidades, competências e conhecimentos na instituição. No contexto acadêmico, a criação de novos conhecimentos científicos dá-se por meio de pesquisa científica. No entanto, outros elementos contribuem diretamente para este processo, conforme relatos dos pesquisadores entrevistados. O ensino, a orientação de trabalhos e contato com os pares foram instâncias recorrentemente mencionadas pelos pesquisadores quando questionados sobre suas atividades de produção. Portanto, a criação do conhecimento científico tem insumos de várias lateralidades, as quais, pode-se dizer, são apoiadas ao longo da execução dos modos de conversão de Nonaka e Takeuchi (1997), nomeadamente socialização, externalização, combinação e internalização. A criação de novos conhecimentos científicos, como processo constituinte do modelo proposto, realimentará o ciclo da gestão do conhecimento científico.

Cabe ressaltar que, no modelo conceitual proposto, a validação não é levada em consideração como processo de gestão do conhecimento, muito embora esteja presente em vários modelos da literatura. No entanto, no contexto da gestão do conhecimento científico, considera-se que o próprio sistema científico exerce naturalmente a função de validação do conhecimento, seja pelo receio do pesquisador em tornar público, mesmo que informalmente, conhecimentos com padrões mínimos de confiabilidade e qualidade, seja pela natural adoção do sistema de peer review por parte das comunidades científicas.

\section{Descrição e detalhamento dos relacionamentos entre os elementos macro e os processos de gestão do conhecimento}

A descrição e o detalhamento dos relacionamentos entre os elementos macro e os processos de gestão do conhecimento do modelo proposto é feita em quatro níveis: relação entre o elemento macro comunidade acadêmica e os processos de gestão do conhecimento; relação entre o elemento macro cultura científica/organizacional e os processos de gestão do conhecimento; relação entre o elemento macro comunicação científica e os processos de gestão do conhecimento, relação entre o elemento macro comunidades científicas e os processos de gestão do conhecimento.

3.1. Comunidade acadêmica e processos de gestão do conhecimento

Embora receba influência externa, a gestão do conhecimento científico, conforme proposição do modelo, ocorre nos limites da comunidade acadêmica, no caso uma universidade.

\subsection{Cultura científica/organizacional e processos de gestão do conhecimento}

A cultura científica influencia os processos de gestão do conhecimento na medida em que a adoção tanto da gestão do conhecimento como um todo, quanto de seus processos individualmente esteja de acordo e contemple as diferenças disciplinares. Assim, embora o modelo proposto apresente uma abordagem geral dos conceitos, a gestão do conhecimento científico em uma universidade - com base no modelo - poderá ocorrer de maneira distinta e gradativamente em cada área do conhecimento. Entretanto, no que diz respeito à cultura organizacional, é necessário levar em conta duas questões. A primeira é que o modelo proposto considera os padrões preconizados pela Iniciativa dos Arquivos Abertos e o Acesso Livre. Como preconiza Lawrence (2001), o acesso livre a resultados de pesquisa pode ser considerado um fator que maximiza o acesso à pesquisa e acelera seus impactos, produtividade, progresso e recompensas. As 
universidades, portanto, devem agir no sentido de incentivar seus membros a adotar uma atitude de disseminação fundamentada nesses padrões. A segunda questão, decorrente da primeira, diz respeito ao desenvolvimento de políticas internas que estimulem um ambiente favorável ao compartilhamento interno de conhecimento, tanto por meios estruturados, quanto por meios informais, conforme mencionado na análise e discussão dos dados das entrevistas (LEITE, 2006), nas quais, de acordo com os sujeitos da pesquisa, barreiras culturais que inibem o compartilhamento devem ser transpostas ou minimizadas.

\subsection{Comunicação científica e processos de gestão do conhecimento}

O modelo de gestão do conhecimento proposto tem nos processos de comunicação científica seu substrato e elemento crucial. A relação entre o elemento macro comunicação científica e os processos de gestão do conhecimento encontra-se descrita em Leite (2006). Cada um dos processos de gestão do conhecimento (identificação, aquisição, armazenagem/organização e criação - este também do ponto de vista dos modos de conversão de Nonaka e Takeuchi) tem nos processos de comunicação científica sua base fundamental. É importante destacar que a comunicação científica informal tem fundamental importância no compartilhamento do conhecimento científico tácito, o qual, por não ser possível de ser compartilhado via sistemas estruturados, tem por base a comunicação informal subjacente às interações sociais. Os processos de gestão do conhecimento do modelo proposto e, sobretudo, o compartilhamento do conhecimento, seja tácito ou explícito, dependem necessariamente da comunicação científica.

\subsection{Comunidades científicas e os processos de gestão do} conhecimento

A principal função do elemento macro comunidades científicas é servir de input para os processos de gestão do conhecimento científico do modelo proposto. Isso se dá, pois pesquisadores-membros da comunidade acadêmica são, individualmente, membros das comunidades científicas. Portanto, ao mesmo tempo que produzem para suas comunidades científicas, estão produzindo necessariamente para sua comunidade acadêmica. Contudo, a questão principal é a seguinte: ao pertencerem a determinadas comunidades científicas, pesquisadoresdocentes têm acesso a fontes de conhecimento, tanto tácito quanto explícito, às quais sua comunidade acadêmica não teria. Como exemplo, é possível citar, no caso do conhecimento explícito, anais de congressos científicos, trocas de trabalhos científicos, livros não disponíveis facilmente, entre outros. No caso do conhecimento científico tácito, é possível citar as trocas de experiências com colegas de instituições nacionais e estrangeiras por meio dos colégios invisíveis, contatos pessoais com pesquisadores renomados, conferências nacionais e internacionais, videoconferências, visitas a outras instituições e laboratórios, realização de estágios pós-doutorais em instituições estrangeiras e outros.

Para cada processo de gestão do conhecimento científico do modelo proposto, as comunidades científicas exercem influências de fora para dentro em uma comunidade acadêmica da seguinte maneira:

3.4.1. Identificação (mapeamento): com base no mapeamento do conhecimento científico interno, podese determinar o que mapear externamente. $O$ mapeamento externo do conhecimento tácito e explícito diz respeito à localização de fontes de conhecimento que estão relacionadas com as competências presentes na comunidade acadêmica. Como exemplo, é possível citar a localização de grupos de pesquisa de outras instituições que se dedicam aos mesmos tópicos, portais de informação científica, sítios pessoais de pesquisadores renomados, bases de dados referenciais e texto completo, listas de discussão, fontes de financiamento e cooperação técnico-científica, calendários de eventos internacionais, entre outros. Todos esses exemplos dizem respeito a itens que estão disponíveis em suas respectivas comunidades científicas.

3.4.2. Aquisição: após serem mapeadas as fontes externas de conhecimento, o processo seguinte, de acordo com o modelo proposto, é a aquisição desses conhecimentos. A aquisição de conhecimento externo dá-se de maneira diversificada. Em relação ao conhecimento científico explícito, as formas tradicionais de aquisição são os serviços bibliotecários, por meio de assinaturas de periódicos científicos, aquisição de material bibliográfico, assinatura de bases de dados e outros serviços. Respeitando-se a questão dos direitos autorais, é possível adquirir muito conhecimento científico externo à comunidade acadêmica publicado na internet e, em uma busca unificada, em repositórios institucionais e temáticos de acesso livre baseados no protocolo OAIPMH (Protocol for Metadata Harvesting). De outra forma, a aquisição de conhecimento externo acontece também com o subsídio oferecido pela instituição para a participação de seus pesquisadores em eventos nacionais 
e internacionais, realização de estágio pós-doutoral, estímulo ao intercâmbio de pesquisadores visitantes e estudantes etc. Tudo isso deve ser acompanhado por uma política institucional que estimule o compartilhamento interno. A aquisição de conhecimentos científicos externos, de acordo com o modelo proposto, é voltada para os espaços das comunidades científicas. Por outro lado, as competências das comunidades acadêmicas se refletem nas comunidades científicas.

\subsubsection{Organização/Armazenagem: o modelo proposto se} insere no contexto do movimento do acesso livre à informação científica, o qual, por sua vez, pressupõe uma arquitetura com características que potencializam não só a organização e a armazenagem dos conteúdos, mas também sua recuperação de maneira mais rápida e mais ampla, se comparado ao modelo tradicional do processo de comunicação científica. Isso ocorre graças aos mecanismos de coleta de metadados. Essa forma de organização e armazenagem deve, necessariamente, utilizar protocolos como o OAI-PMH, que permitem a interoperabilidade dos conteúdos armazenados em arquivos abertos de diferentes áreas do conhecimento em diferentes comunidades científicas (LEITE, 2006).

\subsubsection{Compartilhamento: a relação entre o elemento macro} comunidades científicas e o processo de compartilhamento pode ser analisada a partir de três ângulos. $O$ primeiro é voltado para o compartilhamento do conhecimento em sua vertente tácita, em que as interações sociais e a comunicação informal são imprescindíveis também para o compartilhamento de dentro da comunidade acadêmica para fora. Dessa forma, a comunidade acadêmica tem nas comunidades científicas vetores de compartilhamento. $O$ segundo ângulo de análise diz respeito às publicações formais, as quais têm nos periódicos científicos, livros e capítulos de livros suas principais expressões. Cada pesquisador escolhe os veículos de comunicação apropriados à sua área de conhecimento para compartilhar formalmente o conhecimento que produziu. $\bigcirc$ terceiro e último ângulo de análise se sobrepõe aos dois anteriores, uma vez que contempla tanto a comunicação formal quanto a comunicação informal. A iniciativa dos arquivos abertos e o movimento do acesso livre, sobre os quais se fundamentou também a construção do modelo conceitual proposto, lança mão de uma série de características que permitem ao modelo representar tanto a comunicação formal quanto a informal. Reflete, também, a potencialização da interação entre os pares, permitindo assim o aumento da comunicação informal. Quer se destacar que as duas formas de comunicação são igualmente beneficiadas por recursos próprios da iniciativa dos arquivos abertos e movimento do acesso livre, com especial destaque para a questão da interoperabilidade. A principal questão que liga o elemento macrocomunidades científicas ao processo de gestão do conhecimento compartilhamento é a oportunidade e o aumento da visibilidade, disponibilidade e acessibilidade do conhecimento produzido na comunidade acadêmica.

3.4.5. Criação: Um novo conhecimento científico é criado a partir da interação entre os estoques de conhecimentos existentes e as habilidades e competências dos pesquisadores (LEITE, 2006). Assim, os modos de conversão de Nonaka e Takeuchi (1997) ilustram as relações possíveis entre os conhecimentos registrados e o conhecimento que é relacionado ao que os pesquisadores sabem. No modelo conceitual de gestão do conhecimento proposto, a relação entre o processo de criação e o elemento macrocomunidades científicas dá-se em razão de que o input de conhecimento para a gestão do conhecimento, além de ser interno, no âmbito da própria comunidade acadêmica, é externo, tendo em vista que cada vez mais pesquisadores vinculados a diferentes instituições produzem cada vez mais em colaboração. Além do mais, esse processo tem sido facilitado não só no domínio dos processos formais de comunicação do conhecimento, mas, sobretudo, no domínio da comunicação informal, haja vista a emergência de poderosas ferramentas tecnológicas multimídia. No modelo proposto, consideram-se os mecanismos de interação entre pesquisadores a variedade de tipos de documentos que podem ser armazenados, bem como a integração em um sistema global de repositórios interoperáveis, como recursos que, ao mesmo tempo que possibilitam a busca e recuperação facilitada de informação e conhecimentos externos à comunidade acadêmica em nível global, possibilitam, também, a disponibilidade, acessibilidade e visibilidade de toda a produção científica da própria comunidade. Assim, a conversão do conhecimento conta com as comunidades científicas como fontes de conhecimentos externos à comunidade acadêmica, uma vez que, ao se realizar uma busca no repositório da instituição, é possível incluir nela, simultaneamente, o sistema global de repositórios (LEITE, 2006).

modelo conceitual de gestão do conhecimento científico proposto apresenta o conjunto de elementos macro, os processos e as relações entre eles. A descrição do modelo limitou-se à caracterização dos principais conceitos e as relações entre eles, os elementos macro e 
os processos de gestão do conhecimento científico propriamente dito. Uma vez que a concepção do modelo foi baseada em conceitos - expressos tanto na análise da literatura, quanto na percepção dos entrevistados -, é importante salientar que o modelo não é exaustivo nem tampouco conclusivo. A intenção foi discutir, estruturar e explorar a relação entre conceitos com o fim de delinear um arcabouço teórico inicial para a construção de um corpus de conhecimentos, uma vez que o tópico gestão do conhecimento científico carece de estudos que considerem as especificidades próprias da natureza do conhecimento e do contexto. É importante considerar também que, por uma necessidade de delimitação do objeto de estudo e limitação do tempo disponível para a pesquisa, não foram considerados, no modelo, outros elementos que exerceriam forte influência sobre a gestão do conhecimento científico no contexto acadêmico. Como exemplos, podem ser mencionados a sociedade como um todo e os aspectos da popularização do conhecimento científico para segmentos sociais específicos, a influência direta das agências de fomento sobre a criação do conhecimento científico e por conseqüência a sua gestão e a relação entre universidade e indústria e outros.

\section{CONSIDERAÇÕES FINAIS}

É importante ressaltar que este estudo, ao combinar duas perspectivas teóricas distintas - a comunicação científica e a gestão do conhecimento -, pretendeu, em última análise, servir à construção de uma base teórica para o desenvolvimento de estudos futuros, uma vez que a literatura de ambos os campos está voltada para os seus próprios núcleos. Portanto, o esforço principal, ao propor um modelo conceitual de gestão do conhecimento científico para o ambiente acadêmico tendo por base os processos de comunicação científica, foi delinear e estabelecer a convergência teórica entre os dois tópicos de estudo visando à constituição de um quadro de referência para estudos nesse tópico. Um dos resultados desse esforço foi a estruturação de conceitos que buscam sustentar a abordagem da gestão do conhecimento científico. A exploração dos aspectos do conhecimento tácito na ciência, por exemplo, tanto com o aporte da literatura quanto nas entrevistas, constitui agora passos já percorridos para o aprofundamento da questão em outros estudos e para a formulação e teste de hipóteses.

A gestão do conhecimento científico tendo por base a comunicação científica, aliada a todas as questões exploradas, difere de estudos tradicionais sobre gestão do conhecimento em universidades. Como ressaltado inicialmente, estudos sobre gestão do conhecimento em universidades geralmente abordam o conhecimento científico do ponto de vista do conhecimento organizacional, ou ainda enfocam o desenvolvimento de plataformas tecnológicas, fazem gestão de dados e, quando muito, gestão da informação. Portanto, o caráter teórico do trabalho foi conduzido pela necessidade de se compreenderem os elementos e as relações entre eles que influenciam direta ou indiretamente a gestão do conhecimento científico.

Os processos de gestão do conhecimento científico, no contexto de uma comunidade acadêmica, mesmo que tenham por objetivo oferecer suporte, potencializar e tornar mais eficientes as atividades de pesquisa e ensino na instituição e a integração entre elas - e por conseqüência o estímulo à criação de novos conhecimentos -, sofrem influência externa das comunidades científicas, como sugerem os resultados. Por esta e outras razões, a identificação, aquisição, organização/armazenagem e, sobretudo, o compartilhamento e criação do conhecimento científico, como processos de gestão do conhecimento no contexto de uma universidade, não podem prescindir ou desprezar a interferência de várias lateralidades, tanto internas quanto externas, de caráter cultural, tecnológico ou social. Dessa maneira, uma instituição acadêmica não pode fechar-se em si, pois está inserida em um complexo sistema científico, no qual a influência mais direta e imediata, no que diz respeito aos fenômenos relacionados com o conhecimento, provém das comunidades científicas.

Por fim, entende-se por gestão do conhecimento científico o planejamento e controle de ações (políticas, mecanismos, ferramentas, estratégias e outros) que governam o fluxo do conhecimento científico em sua vertente tácita e explícita, tendo como substratos os processos de comunicação científica com o fim de apoiar e maximizar a criação de novos conhecimentos e o ensino.

Artigo submetido em 17/09/2007 e aceito em 24/10/2007.

\section{REFERÊNCIAS}

BATISTA, Fábio F; COSTA, Sely M. S.; ÁLVARES, Lillian. Gestão do conhecimento: a realização da proposta de Brookes para a Ciência da Informação?. In: ENCONTRO NACIONAL DE PESQUISA EM CIÊNCIA DA INFORMAÇÃO, 8., 2007, Salvador. Anais... Salvador: ANCIB, 2007.

BAUER, M. W.; GASKELL, G. Pesquisa qualitativa com texto, imagem e som: um manual prático. Rio de Janeiro: Vozes, [s.d.]. 516 p. 
BELKIN, N. J. Information concepts for information science. Journal of Documentation, v. 34, n. 1, p. 55-85, Mar. 1978.

BRAGA, G. M. Informação, ciência da informação: breves reflexões em três tempos. Ciência da Informação, v. 24, n. 1, p. 84-88, 1985.

BOUTHILLIER, France; SHEARER, Kathleen. Understanding knowledge management and information management: the need for an empirical perspective. Information Research, v. 8, n. 1, Oct. 2002. Disponível em: < http://informationr.net/ir/8-1/paper 141.html >. Acesso em: 25 maio, 2007.

BROOKES, Bertram. The foundations of information science: part I: philosophical aspects. Journal of Information Science, v. 2, p. 125-133, 1980.

COLLINS, H. M. Tacit knowledge, trust and the $\mathrm{Q}$ of sapphire. Social Studies of Science, n. 31, p.71-85, 2001.

COSTA, S. M. S. The impact of computer usage on scholarly communication amongst academic social scientists. 1999. 302 f. Tese (Doutorado em Ciência da Informação)- Loughborough University, Department of Information Science, Loughborough, Inglaterra, 1999.

CRANE, Diana. Invisible colleges: diffusion of knowledge in scientific communities. Chicago; London: University of Chicago Press, 1972.

GARVEY, W. D.; GRIFFITH, B. C. Scientific communication as a social system. In: COMMUNICATION: the essence of science. London: Pergamon Press, 1979. p. 148-164.

IVES, W.; TORREY, B.; GORDON, C. Knowledge management: an emerging discipline with a long history. Journal of Knowledge Management, v. 1, n. 4, p. 269-274, 1998. Disponível em: <http://www.krii.com/ downloads/km_emerg_discipl.pdf $>$. Acesso em: jan. 2006.

KVALE, S. Interviews: an introduction to qualitative research interviewing. Thousand Oaks: Sage Publications, 1996.

LAGOZE, C.; VAN DE SOMPEL, H. The Open Archives Initiative: building a low-barrier interoperability framework. Disponível em: <www.openarchives.org/documents/jcd12001-oai.pdf>. Acesso em: fev. 2005.

LAWRENCE, S. Free online availability substantially increases a paper's impact. Nature Web Debates, 2001. Disponível em: <http:// www.nature.com/nature/debates/e-access/Articles/lawrence.html>. Acesso em: nov. 2004.

LE COADIC, Yves-François. A ciência da informação. Brasília: Briquet de Lemos, 2004. 124 p.
LEITE, F. C. L. Gestão do conhecimento científico no contexto acadêmico: proposta de um modelo conceitual. 240 f. Dissertação (Mestrado em Ciência da Informação)- Programa de Pós-Graduação em Ciência da Informação, Universidade de Brasília, Brasília, 2006. Disponível em: $<$ http://eprints.rclis.org/archive/00006259/>. Acesso em: 2007.

MEADOWS, A. J. A comunicação científica. Brasília: Briquet de Lemos, 1999. $268 \mathrm{p}$.

NONAKA, I.; TAKEUCHI, H. Criação do conhecimento na empresa: como as empresas japonesas geram a dinâmica da inovação. Rio de Janeiro: Campus, 1997. 358 p.

POLANYI, M. The tacit dimension. London: Routledge e Kegan Paul, 1966.

POPPER, K. R. Conhecimento objetivo: uma abordagem evolucionária. Belo Horizonte: Itatiaia, 1975. 394 p.

PROBST, G.; RAUB, S.; ROMHART, K. Gestão do conhecimento: os elementos construtivos do sucesso. Porto Alegre: Bookman, 2002. 286 p.

SCHEIN, E. H; CALLAGHAN, D. Guia de sobrevivência da cultura corporativa. Rio de Janeiro: J. Olympio, 2001. 191 p.

SMOLIAR, S. W. Interaction management: the next (and necessary) step beyond knowledge management. Business Process Management Journal, v. 9, n. 3, p. 337-353, 2003. Disponível em: < http:// www.emeraldinsight.com/Insight $/$ ViewContentServlet?Filename $=/$ published/emeraldfulltextarticle/pdf/1570090305.pdf> . Acesso em: jan. 2006.

THEUNISSEN, P. Communication: the cornerstone oh knowledge management: making a difference. In: AUSTRALIAN AND NEW ZEALAND COMMUNICATION ASSOCIATION CONFERENCE 2004, 2004, New Zeland. Proceedings... [S.l.: s.n.], 2004.

VAN DE SOMPEL, H.; LAGOZE, C. The Santa Fe Convention of the open archives initiative. D-Lib Magazine, v. 6, n. 3, Feb. 2000. Disponível em: < http://www.dlib.org/dlib/february00/vandesompel-oai/ 02 vandesompel-oai.html>. Acesso em: 20 set. 2006.

WILSON, B. Systems: concepts, methodologies and aplications. Lancaster: John Wiley \& Sons, 1990.

ZIMAN, J. M. A força do conhecimento. Belo Horizonte: Itatiaia, 1981. $380 \mathrm{p}$. 